OPEN ACCESS

Edited by:

Ugo Moretti,

University of Verona, Italy

Reviewed by:

Tanja Mueller,

University of Strathclyde,

United Kingdom

Prince Kasongo Mwila,

Potchefstroom Hospital, South Africa

*Correspondence:

Antoine Pariente

antoine.pariente@u-bordeaux.Fr

Specialty section:

This article was submitted to

Pharmaceutical Medicine and

Outcomes Research,

a section of the journal

Frontiers in Pharmacology

Received: 31 October 2020

Accepted: 29 December 2020

Published: 24 March 2021

Citation:

Létinier L, Ferreira A, Marceron A, Babin $M$, Micallef $J$,

Miremont-Salamé $G$ and Pariente $A$ (2021) Spontaneous Reports of Serious Adverse Drug Reactions

Resulting From Drug-Drug Interactions: An Analysis From the French Pharmacovigilance Database.

Front. Pharmacol. 11:624562.

doi: 10.3389/fphar.2020.624562

\section{Spontaneous Reports of Serious Adverse Drug Reactions Resulting From Drug-Drug Interactions: An Analysis From the French Pharmacovigilance Database}

\author{
Louis Létinier ${ }^{1,2}$, Amandine Ferreira $^{1}$, Alexandre Marceron ${ }^{1}$, Marina Babin ${ }^{3}$, \\ Joëlle Micallef ${ }^{4,5}$, Ghada Miremont-Salamé ${ }^{1,2}$ and Antoine Pariente ${ }^{1,2 *}$ \\ on behalf of the French Network of Pharmacovigilance Centres
}

${ }^{1}$ Univ. Bordeaux, INSERM, BPH, U1219, Team Pharmacoepidemiology, Bordeaux, France, ${ }^{2} \mathrm{CHU}$ de Bordeaux, Pole de Santé Publique, Service de Pharmacologie Médicale, Centre de Pharmacovigilance de Bordeaux, Bordeaux, France, ${ }^{3}$ Service de Pharmacologie Toxicologie et CRPV, CHU, Angers, France, ${ }^{4}$ CRPV Marseille Provence Corse, Service Hospitalo-Universitaire de Pharmacologie Clinique et Pharmacovigilance, Assistance Publique Hôpitaux de Marseille, Marseille, France, ${ }^{5}$ Aix Marseille Université, Institut des Neurosciences des Systèmes, INSERM 1106, Marseille, France

Few data are available on the clinical impact of drug-drug interactions (DDIs). Most of the studies are limited to the analysis of exposure to potential DDI or the targeted impact of the combination of a few drugs or therapeutic classes. The analysis of adverse drug reaction (ADR) reports could be a mean to study generally the adverse effects identified due to a DDI. Our objective was to describe the characteristics of ADRs resulting from DDls reported to the French Pharmacovigilance system and to identify the drugs most often implicated in these ADRs. Considering all ADR reports from January 01, 2012, to December 31, 2016, we identified all cases of ADR resulting from a DDI (DDI-ADRs). We then described these in terms of patients' characteristics, ADR seriousness, drugs involved (two or more per case), and ADR type. Of the 4,027 reports relating to DDI-ADRs, 3,303 were related to serious ADRs. Patients with serious DDI-ADRs had a median age of 76 years (interquartile range: $63-84$ ); 53\% were male. Of all serious DDI-ADRs, $11 \%$ were life-threatening and $8 \%$ fatal. In $36 \%$ of cases, the DDI causing the ADR involved at least three drugs. Overall, 8,424 different drugs were mentioned in the 3,303 serious DDI-ADRs considered. Altogether, drugs from the "antithrombotic agents" subgroup were incriminated in 34\% of serious DDI-ADRs. Antidepressants were the second most represented therapeutic/pharmacological subgroup (5\% of serious DDI-ADRs). Among the 3,843 ADR types reported in the 3,303 serious DDI-ADRs considered, the most frequently represented were hemorrhage $(40 \%$ clinical hemorrhage; $6 \%$ biological hemorrhage), renal failure (8\%), pharmacokinetic alteration (5\%), and cardiac arrhythmias (4\%). Hemorrhagic accidents are still an important part of serious ADRs resulting from DDIs reported in France. The other clinical consequences of DDIs seem less well identified by pharmacovigilance. Moreover, more than one-third of serious DDI-ADRs involved at least three drugs. 


\section{INTRODUCTION}

Exposure to drug-drug interactions (DDIs) is a well-known public health issue (Becker et al., 2008; Létinier et al., 2019). Most of the studies conducted on this subject show an increase in the frequency of potential and clinically relevant DDI over the years, associated with the aging of the population and polymedication (Nobili et al., 2009; Maher et al., 2014; Guthrie et al., 2015). Some pharmacoepidemiological studies have tried to estimate the clinical impact of these DDIs, but it remains difficult given the multiplicity of the drugs involved and the diversity of potential adverse events (Magro et al., 2012; Hennessy et al., 2016). Pharmacovigilance databases of spontaneous reporting can provide complementary information to investigate this impact, as they can allow describing the adverse drug reactions (ADRs) as DDI induced. Despite this, most of the recent publications in the field have not focused on such description but instead on the detection of safety signals regarding unknown DDIs (Jiang et al., 2015; Vilar et al., 2017); in terms of general prescription, we could identify only one study performed in a French region that has shown the reported ADR related to DDI mainly concerning renal failure and hemorrhages (Duval, 2017). The other existing publications were mainly investigating specifically some targeted drugs (Montastruc et al., 2012; Suzuki et al., 2015; Schlit et al., 2017), and none was identified that considered more than two drugs when exploring the consequences of DDIs in terms of ADRs, although this could frequently occur in patients.

In this context, our objective was to describe the characteristics of adverse drug reactions resulting from drug-drug interactions (DDI-ADRs) reported to the French network of Pharmacovigilance Centers and to identify the drugs most often implicated in these. We additionally aimed at describing the DDIs involving more than two drugs.

\section{MATERIALS AND METHODS}

\section{Dataset}

The French Pharmacovigilance system is based on a network of 31 regional centers. In those, ADRs reported by health professionals or patients are evaluated by trained pharmacologists before they are entered into the French Pharmacovigilance Database (BNPV). The ADR declaration is mandatory for physicians and pharmacists but voluntary for patients. In practice, it is especially recommended to report unexpected or serious ADRs. There are different supports and forms of declarations, including an increasingly used national web portal. Each year, around 40,000 ADR reports are collected by this system. The assessment includes a determination of whether ADR is serious and whether it results from a DDI. In this study, we considered all cases of DDI directly coded as such and all cases of ADR, pregnancy exposure, drug misuse or abuse, weaning, or overdoses with at least one drug identified as having caused an interaction. To be serious, an ADR needs to result in death, life-threatening condition, hospitalization (or prolongation of existing hospitalization), persistent or significant disability or incapacity, congenital abnormalities/birth defect, or another significant medical event. If the ADR results from a DDI, the pharmacologist will determine which drugs are suspected of being related to the ADR. Moreover, it may be that more than two drugs are considered suspect, defined here as multiple DDI.

\section{Population and Cases}

Considering all ADR reports entered in the BNPV from January 01, 2012, to December 31, 2016, we identified all cases of serious and nonserious DDI-ADRs. We described general characteristics of the ADR reports considered as DDI and of other ADRs. We then described serious DDI-ADRs in terms of patients' characteristics, ADR seriousness type, drugs involved (two or more per case), and ADR type. Drugs involved were described according to the Anatomical Therapeutic Chemical classification (ATC) Third level (therapeutic/pharmacological subgroups) and Fifth level (chemical substance). In the BNPV, ADRs are coded using the Medical Dictionary for Regulatory Activities (MedDRA) Preferred Terms (PTs). ADR type was consequently studied using Standardized MedDRA Queries (SMQs) or ad hoc defined sets of MedDRA terms when no SMQ was available.

\section{Statistical Analysis}

The general characteristics of patients for whom a DDI-ADR was reported were described, as were those of all patients for whom an ADR was reported, whatever the mechanism. For DDI-ADRs, a description was also performed according to the ADR seriousness (serious vs. nonserious DDI-ADRs). Finally, the description of serious DDI-ADRs was later stratified according to the type of drugs and to the type of ADR.

In addition, a detailed description and visualization of the drug therapeutic/pharmacological subgroups involved in the most frequent DDI-ADR were performed.

Quantitative variables were described in terms of the median and interquartile range (IQR), and qualitative variables were described as proportion, including missing data. Due to the very observational nature of the data and to the expected existence of differences between groups, we did not perform statistical testing and only performed comparative description when studying different groups of patients or serious vs. nonserious DDI-ADRs.

All analysis and visualization were performed using RStudio Version 1.0.143- ${ }^{\circ}$ 2009-2016 RStudio, Inc. 
TABLE 1 | General characteristics of the ADR reports considered as DDI and of the ADR reports not relating to identified DDls entered in the French Pharmacovigilance Database during the 2012-2016 period.

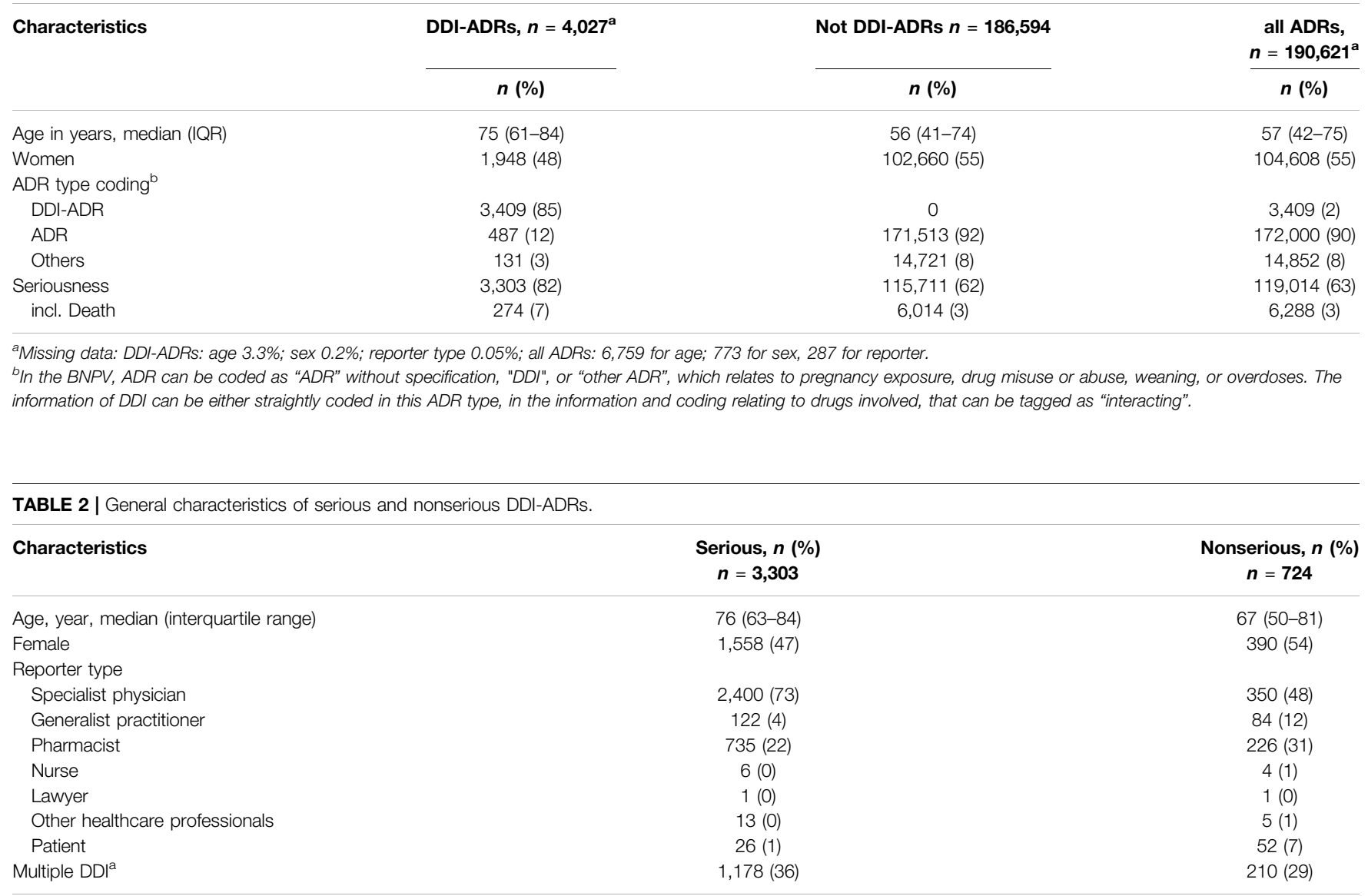

${ }^{a}$ More than two drugs identified as being involved in the interaction.

TABLE 3 | Seriousness criteria of serious DDI-ADR $n=3,303$.

Seriousness Criteria

n (\%)

Death

Life-threatening

Incapacity

Hospitalization

Other significant medical events an ADR was reported in the BNPV over the period, the median age was of 57 years (IQR: 42-75); the proportion of women was $55 \%$.

Among patients for whom a DDI-ADR was reported, those being reported for a serious DDI-ADR appeared older than patients reported for a nonserious one (median age: 76 vs. 67) and to be more likely to be more men (53\%vs. 46\%) (Table 2). Among serious DDI-ADRs reports, DDIs involving more than two drugs appeared more frequent (36\% vs. $29 \%)$.

Of all serious DDI-ADRs, $11 \%$ were life-threatening and $8 \%$ fatal (Table 3). A large part of these cases resulted in or prolonged hospitalization (68\%).

\section{Drugs Involved in Serious Drug-Drug Interactions-Adverse Drug Reactions}

Overall, 8,424 different drugs were mentioned as "suspect" or "interacting" in the 3,303 serious DDI-ADRs considered (median: 2.5 drugs per case). Those most frequently incriminated were fluindione ( $11 \%$ of serious DDI-ADRs), aspirin (8\%), clopidogrel (3\%), warfarin (3\%), and amiodarone (3\%) (Table 4). 
TABLE 4 | Drugs most frequently found among severe cases, ATC fifth and third level $\mathrm{n}=8,484$

\begin{tabular}{lc}
\hline Drugs & $\boldsymbol{n}$ (\%) \\
\hline ATC fifth level & \\
Fluindione & $895(11)$ \\
Aspirin & $690(8)$ \\
Warfarin & $244(3)$ \\
Clopidogrel & $243(3)$ \\
Amiodarone & $224(3)$ \\
Enoxaparin & $186(2)$ \\
Amoxicillin & \\
Furosemide & $168(2)$ \\
Dabigatran & $156(2)$ \\
Escitalopram & $148(2)$ \\
ATC third level & $125(1)$ \\
Antithrombotic agents & \\
Antidepressants & $2,919(34)$ \\
Antipsychotics & $416(5)$ \\
Immunosuppressants & $274(3)$ \\
Antiarrhythmics, classes 1 and 3 & $271(3)$ \\
Opioïds & $233(3)$ \\
Antiinflammatory and antirheumatic products, nonsteroids (NSAIDs) & $216(3)$ \\
Beta-lactam antibacterials, penicillins & $207(2)$ \\
Antiepileptics & $165(2)$ \\
High-ceiling diuretics & $162(2)$ \\
\hline (3) & \\
\hline
\end{tabular}

${ }^{a}$ With or without clavulanic acid.

TABLE 5 | ADR 20 most frequent types among serious cases, $n=3,843^{\mathrm{a}}$.

Adverse reaction type

n (\%)

Hemorrhage

Renal failure

Hemorrhage (biological) ${ }^{\text {b }}$

Pharmacokinetic interaction ${ }^{c}$

Arrhythmia

Fall

Hematologic disorder

Anticholinergic syndrome

Hyperkaliemia

Central nervous system depression

Digestive system disorder

Neurological disorder

Hydroelectrolytic disorders

Drug ineffective

Seizure

Weaning

Rhabdomyolysis

Hepatitis

Death and cardiac arrest

Neuroleptic malignant syndrome

aSome cases contained ADRs belonging to different types.

${ }^{b}$ ADRs corresponding to MedDRA SMQ: "Hemorrhage laboratory terms" (a change in INR, PTT, or clotting factors). The list of corresponding PTs can be viewed at: http:// bioportal. bioontology. org/ontologies/MEDDRA? $p=$ classes\&conceptid=20000040

'Increase or decrease in plasma concentration.

Altogether, drugs from the antithrombotic agents subgroup were incriminated in $34 \%$ of serious DDI-ADRs. Antidepressants were the second most represented therapeutic/pharmacological subgroup (5\% of serious DDI-ADRs) (Table 4).
TABLE 6 | ADR 10 most frequent types among fatal cases, $n=274^{\mathrm{a}}$.

\begin{tabular}{|c|c|}
\hline Adverse reaction type & $n(\%)$ \\
\hline Hemorrhage & $209(76)$ \\
\hline Renal failure & $21(8)$ \\
\hline Cardiac arrest & $20(7)$ \\
\hline Pharmacokinetic interaction ${ }^{\mathrm{b}}$ & $12(4)$ \\
\hline Hematologic disorder & $10(4)$ \\
\hline Central nervous system depression & $9(3)$ \\
\hline Arrhythmia & $7(3)$ \\
\hline Nonhemorrhagic shock & $7(3)$ \\
\hline Digestive system disorder & $6(2)$ \\
\hline Hyperkaliemia & $5(2)$ \\
\hline
\end{tabular}

${ }^{a}$ Some cases contained ADRs belonging to different types.

${ }^{b}$ Increase or decrease in plasma concentration.

TABLE 7 | ATC third level of drugs most frequently found among serious hemorrhage cases (total of 3,648 drugs).

\begin{tabular}{lc} 
ATC 3 & $\boldsymbol{n}(\%)$ \\
\hline Antithrombotic agents & $2,537(70)$ \\
Antidepressants & $179(5)$ \\
Antiarrhythmics, classes 1 and 3 & $142(4)$ \\
Beta-lactam antibacterials, penicillins & $115(3)$ \\
Antiinflammatory and antirheumatic products, nonsteroids & $88(2)$ \\
Lipid modifying agents, plain & $62(2)$ \\
Quinolone antibacterials & $60(2)$ \\
Macrolides, lincosamides, and streptogramins & $50(1)$ \\
Other beta-lactam antibacterials & $48(1)$ \\
Corticosteroids for systemic use, plain & $46(1)$
\end{tabular}

\section{Clinical Consequences of Drug-Drug Interaction and Types of Adverse Drug Reaction}

Across the 3,843 ADR types reported in the 3,303 serious DDIADRs considered, the most frequently represented were hemorrhage (40\%; plus biological hemorrhage, 6\%), renal failure (8\%), pharmacokinetic alteration (5\%), and cardiac arrhythmias (4\%) (Table 5). Hemorrhage accounted for $76 \%$ of the deaths associated with DDI (Table 6). We, therefore, found an average of 1.16 ADR types per serious DDI-ADRs. Pharmacovigilance declarations may indeed contain more than one ADR type.

\section{Drug-Drug Interaction-Adverse Drug Reaction Resulting in Hemorrhages}

Antithrombotic agents were predominant (70\%) among the 3,648 drugs involved in the 1,529 serious DDI-ADRs resulting in hemorrhage. The other main classes represented were antidepressants (5\%), antiarrhythmics (4\%), and penicillins (3\%) (Table 7). Among these cases of hemorrhage, 1,518 (99\%) concern at least one antithrombotic agent and the majority of DDIs involved two antithrombotic agents $(n=856,56 \%)$ (Figure 1). The other frequently encountered associations concerned antithrombotics + antidepressants $(n=126$, 


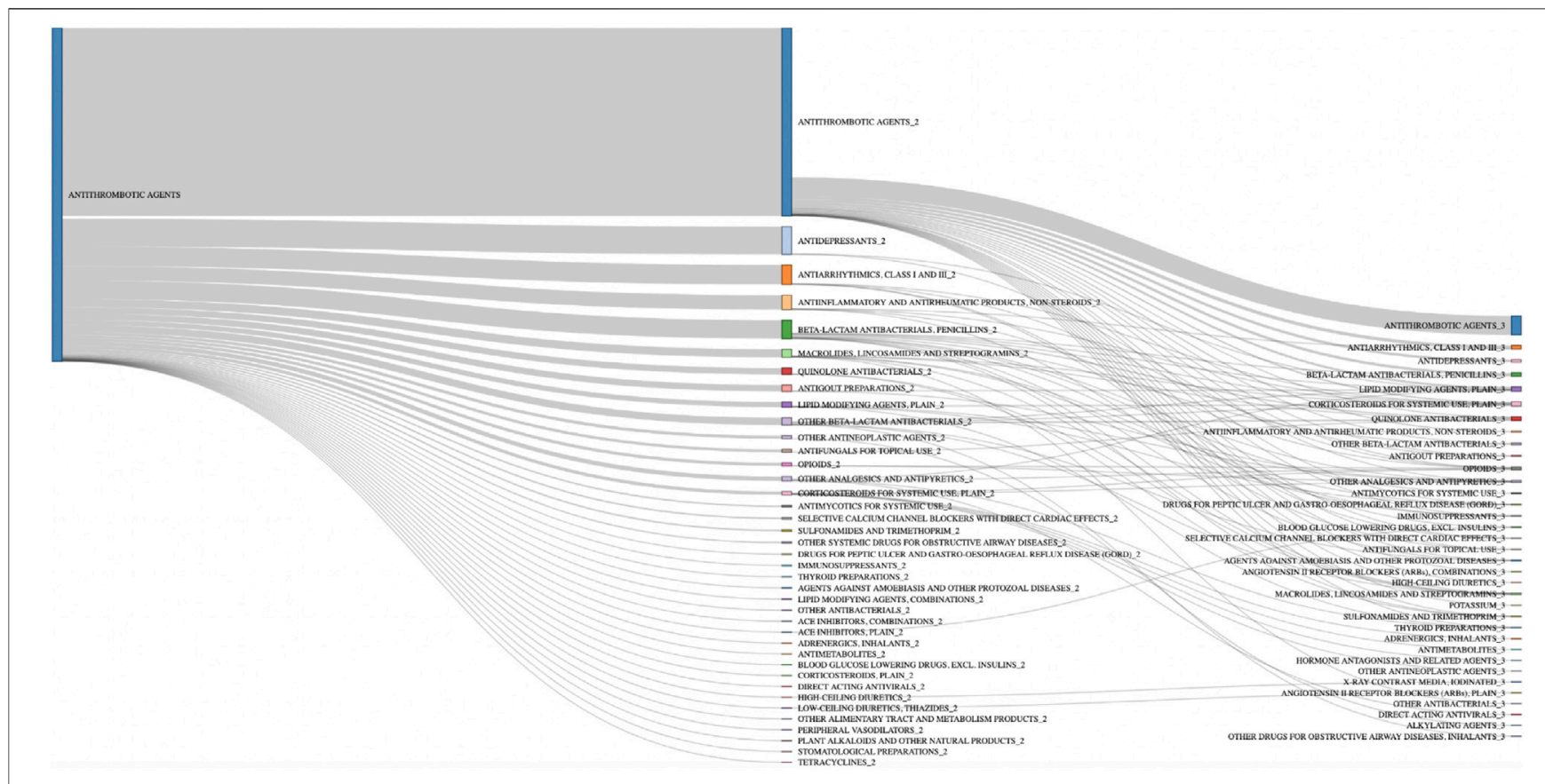

FIGURE 1 | Drug therapeutic/pharmacological subgroup combinations involved in hemorrhage cases with antithrombotic agents $n=1,518$.

TABLE 8 | Drug therapeutic/pharmacological subgroup combinations involved in hemorrhage cases without antithrombotic agents, $n=11$

ATC 3

All other therapeutic products + drugs for constipation

Antidepressants + drugs for peptic ulcer and gastroesophageal

Antifungals for systemic use + corticosteroids for systemic use, plain + immunosuppressants + immunosuppressants

8.2\%; antithrombotics + escitalopram, $n=42$ cases, $2.8 \%$ ), antithrombotics + antiarrhytmics $(n=89,5.8 \%)$, antithrombotics + penicillins $(\mathrm{n}=84,5.5 \%)$, and antithrombotics + NSAIDs $(\mathrm{n}=66,4.3 \%)$. Finally, 254 DDI-ADRs resulting in hemorrhage (17\%) involved more than two drugs; the most frequent association in those involved the combination of three $(n=86,5.6 \%)$.

Among the 11 cases without antithrombotic agents, 5 cases were found with an antidepressant, including 3 cases with an NSAID (Table 8).

\section{DISCUSSION}

ADRs identified as resulting from DDIs constituted only $2.1 \%$ of the spontaneous reports recorded in the French Pharmacovigilance Database from 2012 to 2016. The reports of DDI-ADR concerned men and older patients more than the reports of $\mathrm{ADR}$ in general.
More than $80 \%$ of reported DDI-ADRs were serious and $7 \%$ were fatal; this fatality rate was twice higher than that observed overall for all the reported ADRs. The drugs most frequently incriminated in the occurrence of serious DDI-ADRs were antithrombotics (more than a third) and antidepressants (5\%); a third of the informed DDIs for those serious DDI-ADRs involved at least three interacting drugs. In terms of clinical consequence, hemorrhage constituted around half of the provoked serious adverse reactions and two-thirds of the fatal ones. The DDIs involved in their occurrence resulted mostly from the association of two antithrombotics or from the association of antithrombotics and antidepressants.

The proportion of spontaneously reported ADRs resulting from a DDI is $2.1 \%$ in this study. As compared to the literature where it can range from 5 to $20 \%$, this appears low (Peyriere et al., 2003; Leone et al., 2010; Bénard-Laribière et al., 2015). Our finding could either result from an under coding of interactions and DDI-ADRs in French Pharmacovigilance Centers or from a specific underreporting of these ADRs in 
France. The latter hypothesis could imply that DDI-ADR are less considered when attributed to the drug than to the prescriber and thus would be less needed to be reported in terms of safety evaluation of the drugs. In the same perspective, it could also be that prescribers would be less prone to report such events for which they would potentially be responsible, a barrier to reporting that has already been well identified in the literature (Mirošević Skvrce et al., 2011). This would however paradoxically be less true for serious DDI-ADR than for nonserious one, as the seriousness rate appears high for DDI-ADRs compared to all reported ADRs. However, in that situation, often implying hospitalization, the reporter could be more likely to be the clinician responsible for the ADR management rather than the prescriber.

This study showed that the majority of serious DDI-ADRs were related to antithrombotic agents (anticoagulant or antiplatelet drugs), consistent with the literature which clearly identifies hemorrhage as a well-known consequence of a serious DDI (Pirmohamed et al., 2004; Leone et al., 2010). Even with its intrinsic limitation of underreporting, this study confirmed, in this perspective, that this issue is still involving the occurrence of a very large number of serious hemorrhagic accidents due to DDI. Additionally, it highlighted on this subject that $30 \%$ of the drugs involved in these hemorrhages are not antithrombotic agents and allowed observing that the association of antithrombotic plus antidepressants was responsible for a significant proportion of serious cases of hemorrhage relating to DDIs. This interaction is well known and led to a warning in the French recommendations regarding DDIs (Interactions médicamenteuses, 2017). However, this recommendation nowadays considers the DDI involving this combination to be a Level 1 (over four) in terms of associated risk. The results make a plea for reconsidering this classification. If its extent was not expected, the finding of this DDI is consistent with the literature in which this DDI is highlighted (Schelleman et al., 2011) even its mechanism remains only partly elucidated, at least for its pharmacokinetic aspects (Sansone and Sansone, 2009). Some studies suggested that citalopram is possibly one of the antidepressants with the lowest risk of interaction because it is a weak enzyme inhibitor of CYP P450 (Brøsen and Naranjo, 2001; Sansone and Sansone, 2009). However, in our study, its derivative, escitalopram appeared to constitute the antidepressant most often involved in bleeding events; however, this finding is only related to its wide use in France. Since Selective Serotonin Reuptake Inhibitors (SSRIs) can cause thrombocytopenia and bleeding (by lowering platelet serotonin and inhibiting subsequent serotonin-mediated platelet activation), this may actually be a pharmacodynamic rather than a pharmacokinetic interaction (Laporte et al., 2017). Another interesting result is that fluindione was the drug most often involved in hemorrhage; in 11\% of cases for which it was reported, amoxicillin was the other drug suspected. A similar association was found between warfarin and amoxicillin in $15 \%$ of cases for which warfarin was reported. If publications exist (Farnier et al., 2016), responsibility for an interaction between these drugs or with the underlying infection is still debated (Zhang et al., 2011).
Although hemorrhage is one of the most expected serious sideeffects after a DDI, their overrepresentation in this study suggests that other DDIs could be less easily identifiable by healthcare professionals. For instance, renal failure was the second clinical consequence observed in this study but represented only $8 \%$ of cases. This could be attributed to the fact that this condition can find multiple etiologies other than iatrogenic etiology and would thus be less likely to be identified as such when it is the case (Perazella, 2018). The same goes for arrhythmias which only represented $4 \%$ of reported DDI-ADRs, although it constituted one of the most expected ADR resulting from DDI in the literature (Dechanont et al., 2014; Holm et al., 2014; Létinier et al., 2019).

The main strength of our study was focusing on actual clinical consequences of DDI and not on potential interactions. Another strength of our work was that $99 \%$ of serious cases were reported by healthcare professionals and $100 \%$ of cases were evaluated by a pharmacologist. The use of the BNPV is particularly interesting for more in-depth studies of such a complex pharmacological problem as DDI. Finally, our study was not limited to the interactions involving two drugs. In fact, more than a third of the cases found included at least three suspect drugs; this result advocates for the conduct of further evaluations regarding these more complex situations of drug interactions. However, our descriptive study did not allow us to go further in the analysis of multiple interactions. We are working on the development of specific analytical methods that we will present in future work. The main limitation of our study relates to the underreporting inherent in pharmacovigilance databases (Hazell and Shakir, 2006), which limits the representativeness of our results. Our results suggest that this undernotification would be even stronger for DDI-ADR than for ADR in general. Indeed, it is possible that healthcare professionals are reluctant to report ADR-DDI when it is a well-known mechanism. However, we observe a very high proportion of hemorrhages that are well-known DDIs, so it could be that the difficulty of diagnosing less known DDIs explains this undernotification. Finally, our data were only French and we cannot exclude specific national features in our results. Despite the specificity of the French Pharmacovigilance system, the lessons of this study might be transposed to most countries with similar drug use.

\section{CONCLUSION}

Hemorrhagic accidents are still accounted for as an important part of ADRs resulting from DDIs reported in France and did not only concern antithrombotic agents. Despite being known in theory, these interactions still seem to be present in current practice. In addition, most of the interactions leading to serious cases are not referenced as a high level of severity interactions in the various DDI reference documents. Taken together with the finding that more than one-third of serious DDI-ADRs involved at least three drugs and are more difficult to identify, this highlights the need for tools dedicated to professionals and patients for better management of DDIs and improving the reporting of DDI-ADRs. 


\section{DATA AVAILABILITY STATEMENT}

The data analyzed in this study were subject to the following licenses/ restrictions: The French Pharmacovigilance Database is reserved for use by competent authorities. Requests to access these datasets should be directed to Mehdi.BENKEBIL@ansm.sante.fr.

\section{ETHICS STATEMENT}

The studies involving human participants were reviewed and approved by the French Medicines Agency (Agence Nationale de Sécurité du Médicament et des Produits de Santé, ANSM). Written informed consent from the participants' legal guardian/next of kin was not required to participate in this study in accordance with the national legislation and the institutional requirements

\section{REFERENCES}

Becker, M. L., Visser, L. E., Van Gelder, T., Hofman, A., and Stricker, B. H. C. (2008). Increasing exposure to drug-drug interactions between 1992 and 2005 in people aged $\geq 55$ years. Drugs \& Aging 25, 145-152. doi:10.2165/00002512200825020-00006

Bénard-Laribière, A., Miremont-Salamé, G., Pérault-Pochat, M.-C., Noize, P., and Haramburu, F. (2015). The EMIR Study Group on behalf of the French network of pharmacovigilance centresIncidence of hospital admissions due to adverse drug reactions in France: the EMIR study. Fundam. Clin. Pharmacol. 29, 106-111. doi:10.1111/fcp.12088

Brøsen, K., and Naranjo, C. A. (2001). Review of pharmacokinetic and pharmacodynamic interaction studies with citalopram. Eur. Neuropsychopharmacol. 11, 275-283. doi:10.1016/s0924-977x(01)00101-8

Dechanont, S., Maphanta, S., Butthum, B., and Kongkaew, C. (2014). Hospital admissions/visits associated with drug-drug interactions: a systematic review and meta-analysis. Pharmacoepidemiol. Drug Saf. 23, 489-497. doi:10.1002/ pds. 3592

Duval, M. (2017). Intérêt et importance de la pharmacovigilance dans le suivi et l'amélioration des données sur les interactions médicamenteuses: caractérisation de plus de 700 cas de diagnostics iatrogènes médicamenteux analysés par, 93. Marseille, France: Centre régional de Pharmacovigilance Marseille-ProvenceCorse.

Farnier, E., Charhon, N., Papillon, L., and Tod, M. (2016). Interaction entre amoxicilline/acide clavulanique et fluindione : à propos de deux cas. Thérapie. doi:10.2515/therapie/2015059

Guthrie, B., Makubate, B., Hernandez-Santiago, V., and Dreischulte, T. (2015). The rising tide of polypharmacy and drug-drug interactions: population database analysis 1995-2010. BMC Med. 13, 74. doi:10.1186/s12916-015-0322-7

Hazell, L., and Shakir, S. A. W. (2006). Under-reporting of adverse drug reactions: a systematic review. Drug Saf. 29, 385-396. doi:10.2165/00002018-20062905000003

Hennessy, S., Leonard, C., Gagne, J., Flory, J., Han, X., Brensinger, C., et al. (2016). Pharmacoepidemiologic methods for studying the health effects of drug-drug interactions. Clin. Pharmacol. Ther. 99:92-100. doi:10.1002/cpt.277

Holm, J., Eiermann, B., Eliasson, E., and Mannheimer, B. (2014). A limited number of prescribed drugs account for the great majority of drug-drug interactions. Eur. J. Clin. Pharmacol. 70, 1375-1383. doi:10.1007/s00228014-1745-3

Interactions médicamenteuses (2017). ANSM: Agence nationale de sécurité $d u$ médicament et des produits de santé Available at: http://ansm.sante.fr/Dossiers/ Interactions-medicamenteuses/Interactions-medicamenteuses/(offset)/0.

Jiang, G., Liu, H., Solbrig, H. R., and Chute, C. G. (2015). Mining severe drug-drug interaction adverse events using Semantic Web technologies: a case study. BioData Min 8. doi:10.1186/s13040-015-0044-6

\section{AUTHOR CONTRIBUTIONS}

LL and AP planned and performed the study and drafted the manuscript. GM, MB, and JM helped in designing the study, interpreting the results, and revising the manuscript. AF and AM participated in the statistical analyses and results interpretation. All authors contributed to the preparation of the final version of this manuscript.

\section{ACKNOWLEDGMENTS}

The authors would like to thank all members of the French Network of Pharmacovigilance Centres and the Agence Nationale de Sécurité du Médicament et des Produits de Santé (ANSM) for the availability and the accuracy of the data.

Laporte, S., Chapelle, C., Caillet, P., Beyens, M.-N., Bellet, F., Delavenne, X., et al. (2017). Bleeding risk under selective serotonin reuptake inhibitor (SSRI) antidepressants: a meta-analysis of observational studies. Pharmacol. Res. 118, 19-32. doi:10.1016/j.phrs.2016.08.017

Leone, R., Magro, L., Moretti, U., Cutroneo, P., Moschini, M., Motola, D., et al. (2010). Identifying adverse drug reactions associated with drug-drug interactions. Drug Saf. 33, 667-675. doi:10.2165/11534400-000000000-00000

Létinier, L., Cossin, S., Mansiaux, Y., Arnaud, M., Salvo, F., Bezin, J., et al. (2019). Risk of drug-drug interactions in out-hospital drug dispensings in France: results from the DRUG-drug interaction prevalence study. Front. Pharmacol. 10, 265. doi:10.3389/fphar.2019.00265

Magro, L., Moretti, U., and Leone, R. (2012). Epidemiology and characteristics of adverse drug reactions caused by drug-drug interactions. Expert Opin. Drug Saf. 11, 83-94. doi:10.1517/14740338.2012.631910

Maher, R. L., Hanlon, J. T., and Hajjar, E. R. (2014). Clinical consequences of polypharmacy in elderly. Expert Opin. Drug Saf. 13. doi:10.1517/14740338. 2013.827660

Mirošević Skvrce, N., Macolić Šarinić, V., Mucalo, I., Krnić, D., Božina, N., and Tomić, S. (2011). Adverse drug reactions caused by drug-drug interactions reported to Croatian Agency for Medicinal Products and Medical Devices: a retrospective observational study. Croat. Med. J. 52, 604-614. doi:10.3325/cmj. 2011.52.604

Montastruc, F., Sommet, A., Bondon-Guitton, E., Durrieu, G., Bui, E., Bagheri, H., et al. (2012). The importance of drug-drug interactions as a cause of adverse drug reactions: a pharmacovigilance study of serotoninergic reuptake inhibitors in France. Eur. J. Clin. Pharmacol. 68, 767-775. doi:10.1007/ s00228-011-1156-7

Nobili, A., Pasina, L., Tettamanti, M., Lucca, U., Riva, E., Marzona, I., et al. (2009). Potentially severe drug interactions in elderly outpatients: results of an observational study of an administrative prescription database. J. Clin. Pharm. Ther. 34, 377-386. doi:10.1111/j.1365-2710.2009.01021.x

Perazella, M. A. (2018). Pharmacology behind common drug nephrotoxicities. CJASN 13, 1897-1908. doi:10.2215/cjn.00150118

Peyriere, H., Cassan, S., Floutard, E., Riviere, S., Blayac, J.-P., Hillaire-Buys, D., et al. (2003). Adverse drug events associated with hospital admission. Ann. Pharmacother. 37, 5-11. doi:10.1345/aph.1c126

Pirmohamed, M., James, S., Meakin, S., Green, C., Scott, A. K., Walley, T. J., et al. (2004). Adverse drug reactions as cause of admission to hospital: prospective analysis of 18820 patients. BMJ 329, 15-19. doi:10.1136/bmj.329.7456.15

Sansone, R. A., and Sansone, L. A. (2009). Warfarin and antidepressants: happiness without hemorrhaging. Psychiatry 6, 24.

Schelleman, H., Brensinger, C. M., Bilker, W. B., and Hennessy, S. (2011). Antidepressant-warfarin interaction and associated gastrointestinal bleeding risk in a case-control study. PLoS One 6. doi:10.1371/journal.pone.0021447

Schlit, A., Delaunois, A., Colomar, A., Claudio, B., Cariolato, L., Boev, R., et al. (2017). Risk of QT prolongation and torsade de pointes associated with 
exposure to hydroxyzine: re-evaluation of an established drug. Pharmacol. Res. Perspect. 5, e00309. doi:10.1002/prp2.309

Suzuki, A., Yuen, N. A., Ilic, K., Miller, R. T., Reese, M. J., Brown, H. R., et al. (2015). Comedications alter drug-induced liver injury reporting frequency: data mining in the WHO VigiBase. Regul. Toxicol. Pharmacol. 72, 481-490. doi:10.1016/j. yrtph.2015.05.004

Vilar, S., Friedman, C., and Hripcsak, G. (2017). Detection of drug-drug interactions through data mining studies using clinical sources, scientific literature and social media. Brief Bioinform 19, 863-877. doi:10.1093/bib/bbx010

Zhang, Q., Simoneau, G., Verstuyft, C., Drouet, L., Bal dit Sollier, C., Alvarez, J.-C., et al. (2011). Amoxicillin/clavulanic acid-warfarin drug interaction: a randomized controlled trial. Br. J. Clin. Pharmacol. 71, 232-236. doi:10. $1111 / \mathrm{j} .1365-2125.2010 .03824 . \mathrm{x}$
Conflict of Interest: LL is the cofounder of the SYNAPSE Medicine start-up, which is a mobile app designed to help health professionals and patients identify drug-drug interactions within prescriptions. LL and AP are coinventors of a patent on "device and method for generating a drug database," which is used by the SYNAPSE Medicine mobile application.

Copyright $\odot 2021$ Létinier, Ferreira, Marceron, Babin, Micallef, Miremont-Salamé and Pariente. This is an open-access article distributed under the terms of the Creative Commons Attribution License (CC BY). The use, distribution or reproduction in other forums is permitted, provided the original author $(s)$ and the copyright owner(s) are credited and that the original publication in this journal is cited, in accordance with accepted academic practice. No use, distribution or reproduction is permitted which does not comply with these terms. 